\title{
The effects of tegmental lesions on the reward properties of septal stimulation'
}

Bernard B. Sehiff NORTHWESTERN UNIVERSITY

\begin{abstract}
Lesions in the ventral tegmentum attenuated, and in some cases completely destroyed the reward properties of septal self-stimulation. Lesions in the dorsal mesencephalon (which included damage to the dorsal tegmentum) did not have this effect. It is likely that it was the locus of the tegmental damage rather than the extent which was important. Also, the attenuation in self stimulation behavior was not due to a general depression in activity, or ability to perform the response consequent to the lesion.
\end{abstract}

\section{Problem}

There are several studies in the literature which attempt to describe the functional dependencies between the various reward structures in the rat's brain. Ward $(1960,1961)$ has studied the effects of fornix, septal and amygdaloid lesions on the reward properties of electrical stimulation in the ventral tegmentum. Lesions in these structures did not destroy or, apparently, greatly impair the motivational properties of the stimulation. Anatomical (Nauta, 1956; Nauta \& Kuypers, 1958) and physiological (Milner, 1957) considerations suggest that the tegmental reticular formation might be involved in mediating the properties of stimulation in the other reward structures. More specifically, demonstrations of the functional organization of the midbrain tegmentum with respect to electrical brain stimulation suggest that the ventral tegmentum would be so involved. Thus, in the present study the effects of lesions in the dorsal and ventral tegmentum on the reward properties of septal stimulation were studied.

\section{Merhod}

The apparatus was a conventional skinner box, equipped so as to deliver current to the rats' brains for the duration of the depression of the bar, or for a maximum of $.5 \mathrm{sec}$. Also, each bar-press was registered on a counter. The subjects were 45 naive male albino rats. They had bipolar nichrome electrodes implanted in the septal area.

The experiment consisted of two phases; a preoperative phase and a postoperative phase. The preoperative phase began with 3 days of operant testing. This was followed, after determination of a suitable stimulating current, by successive days of self-stimulation which continued for each animal until it had achieved a stable level of performance. The criterion for stability was, that on 3 consecutive days no score deviated from the median score by more than $25 \%$. Where the 3 scores increased successively, a more stringent criterion of $15 \%$ was used.

After the self-stimulation rates were established the animals were assigned to one of 3 groups. In one group (the Dorsal group) electrolytic lesions were placed stereotaxically in the dorsal mesencephalon. In another group (the Ventral group) they were placed in the ventral tegmentum. The third group underwent a sham operation in which no lesions were made.

The animals were allowed 3 days of recovery before beginning the postoperative phase of testing. This phase was divided into 2 segments. Each segment was composed of successive periods of operant and self-stimulation testing, of 3 and 5 days duration respectively. The second segment began 2 weeks after the operations. All test sessions were $30 \mathrm{~min}$. long.

\section{Results}

Only 21 animals completed the experiment. There were 7 animals in each group. The other 24 either died consequent to the operation, or were discarded because they did not self-stimulate preoperatively.

The ventral tegmental lesions greatly depressed self-stimulation rates (see Fig. 1). A comparison between the Control and Ventral groups yielded a significant Group by Phase interaction $(\mathrm{p}<.05) .{ }^{2}$ Also, an evaluation of the postoperative changes in stimulation rate, using the Mann Whitney U statistic showed that the difference between the Ventral and Control groups had a probability of .019 .

There were no other significant differences between these 2 groups. In 4 of the 7 animals in the Ventral group, the postoperative self-stimulation rates were the same as the postoperative operant rates, suggesting that for these animals the electric stimulus had become neutral. These animals also showed no recovery over time. Two other animals continued to bar press post-

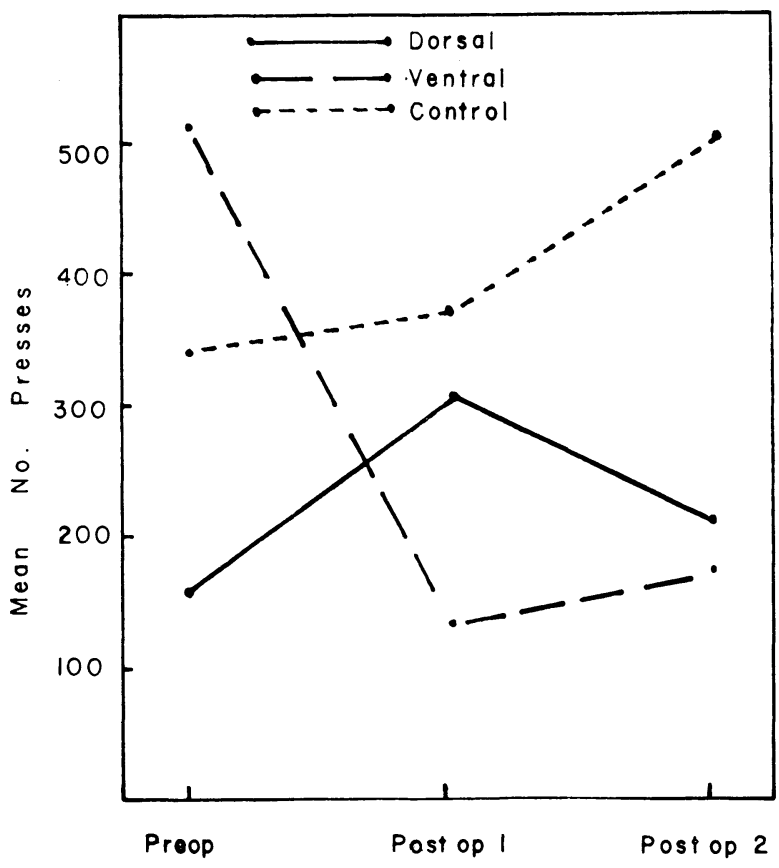

Fig. 1. Mean number of presses for stimulation per half hour for each group at each stage of the experiment. 
operatively, but at greatly reduced rates. The change in rates for these animals was from preoperative scores of 1451 and 787 to postoperative scores of 187 and 308 respectively. These animals improved slightly in the second postoperative segment. The seventh animal did not show the decrease. The Dorsal and Control groups showed no comparable attenuation in self-stimulation behavior, and did not differ statistically from each other.

The operant rates of all groups increased postoperatively. The analysis of variance comparing the 3 groups yielded a significant $F(p<.01)$ for the Phase effect (i.e., preoperative compared with postoperative scores), however there was no significant Group effect or Group interaction effects. The increase in operant rate is most easily attributable to secondary reinforcers present in the situation consequent to the preoperative self-stimulation.

Figure 2 is a composite drawing showing representative lesions for both the Dorsal and Ventral groups. The ventral lesions extended, generally, from the level of the posterior commissure, caudally to the red nuclear level. The dorsal lesions were somewhat more anteriorly place. There was variability in the size and placement of the lesions, however there were no demonstrable relationships within the groups between

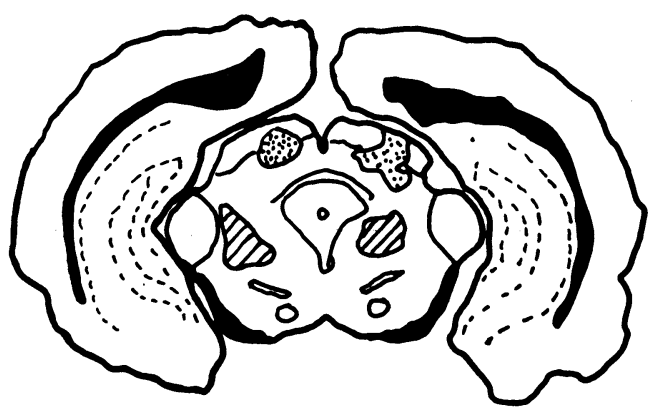

Fig. 2. Composite drawing showing representative lesions of the Dorsal and Ventral groups. The dorsal lesions were aimed at points $5.5 \mathrm{~mm}$ posterior to bregma, $1.5 \mathrm{~mm}$ lateral to the midline and $5 \mathrm{~mm}$ below the skull. The ventral lesions were aimed at points $5.5 \mathrm{~mm}$ posterior to bregma, $1.5 \mathrm{~mm}$ lateral to the midline and 6.5 below the skull. extents or loci of the lesions and their effects. Rank order correlations between the extent of the ventral lesions (estimated by counting the number of units of .1 in graph paper which were covered by the maximal extent of the lesions) and the percentage changes and absolute changes in bar-pressing rates were far from significant (rho being -.07 and .18 respectively).

\section{Diseussion}

The integrity of the ventral tegmentum appears to be necessary to sustain septal self-stimulation. And the operant data indicate that the decrement in self-stimulation rate is not due to deficits in the animals' ability to perform the motor response, or to some generalized depression in activity. The Ventral group showed an increase in operant rate comparable to the other groups.

Because the dorsal lesions involved relatively less tegmental tissue, it is difficult to ascertain that the effect is restricted to the ventral tegmentum. However, the fact that there was no mass action within the Ventral group increases our confidence that the dorsal lesions did serve as a control for locus of tegmental damage.

In conclusion, the ventral tegmental lesions destroyed, or greatly attenuated the reward properties of septal stimulation. It is likely, though not conclusive in this report, that the effect is due to the locus rather than the extent of the tegmental damage.

References

MILNER, P. M. The cell assembly: Mark 11. Psychol. Rev., 1957, 64, 242-252.

NAUTA, W. J. H. An experimental study of the fornix system in the rat. J. comp. Neurol., 1956, 104, 247-292.

NAUTA, W. J. F., \& KUY PERS, H. G. J. M. Some ascending pathways in the brain stem. In H. H. Jaspers, L. D. Proctor, R. S. Knightson, W. C. Hoshay, \& R. T. Costelle (Eds.), Reticular Formation of the brain. Boston: Little, Brown and Co., 1958, 3-30.

WARD,. H. P. Basal tegmental self-stimulation after septal ablation in rats. Arch. Neurol., 1960, 3, 158-162.

WARD, H. P. Tegmental self-stimulation after amygdaloid ablation. Arch. Neurol., $1961,4,657$.

\section{Notes}

1. This report is based on a thesis which was submitted in partial fulfillment of the requirements for a $M$. S. degree at Northwestern University. I thank Dr. S. E. Glickman for direction and assistance. 2. The phase main effect in the analysis of variance was not interpretable because of the preoperative differences between the groups. The large differences, however, did not represent consistent differences between the groups, but rather resulted from a few deviant subjects. Reducing the number of subjects to 5 per group yielded groups which were comparable in their preoperative self-stimulation rates. The relationships between these groups postoperatively were virtually identical with those shown in Figure 1. 